\title{
Collective effects of breakfast consumption and exercise on subsequent postprandial metabolism, appetite and energy balance
}

\author{
J. T. Gonzalez, R. C. Veasey and E. J. Stevenson \\ Brain, Performance and Nutrition Research Centre, School of Life Sciences, Northumbria University, \\ Newcastle, NE1 8ST, UK
}

Consumption of breakfast prior to a test drink, can improve glucose tolerance and attenuate feelings of hunger, reducing subsequent energy intake ${ }^{(1)}$. Acute exercise can also influence glucose uptake ${ }^{(2)}$ and at least transiently, appetite ${ }^{(3)}$. Exercise studies are predominantly performed in the fasted state, limiting the application for typical daily living where exercise may be performed postprandially.

This study aimed to investigate the combined effects of breakfast consumption and exercise on the metabolic and appetite responses later in the day.

In a randomised, crossover design, 12 healthy, physically active males (mean age $23.2 \pm 4.3$ years, body mass $77.2 \pm 5.3 \mathrm{~kg}$, BMI $24.5 \pm 2.0 \mathrm{~kg} / \mathrm{m}^{2}$ and $\mathrm{V}_{\text {O2peak }} 53 \pm 6 \mathrm{ml} / \mathrm{kg} / \mathrm{min}$ ), completed 4 main trials consisting of fasting and rest (FR), breakfast consumption and rest (BR), fasting and exercise (FE) and breakfast consumption and exercise (BE). Exercise consisted of running at $60 \% \mathrm{~V}_{\mathrm{O} 2 \mathrm{peak}}$ until $\sim 2400 \mathrm{~kJ}$ had been expended. A chocolate milkshake test drink was then consumed, followed by a 90 min postprandial period preceding an ad libitum lunch to assess energy intake. Blood samples were used to determine glucose, serum insulin and NEFA concentrations along with plasma glucagon-like peptide-1 $1_{7-36}\left(\mathrm{GLP}_{1-36}\right)$ concentrations. Expired gas was sampled to estimate energy expenditure and substrate metabolism via indirect calorimetry and visual analogue scales were used to assess appetite sensations. Due to difficulties with blood collection in some participants, data for GLP-1 $1_{7-36}$ are presented from 10 participants and for all other blood analytes from 11 participants.

Comparisons between trials were made by repeated measures ANOVA with Holm-Bonferonni post-hoc step-wise adjustment. Significance was accepted at $P<0.05$.

Following consumption of the test drink, peak blood glucose concentration was greater in the BE trial compared to both FE and BR ( $P=0.06$ and $P=0.03$, respectively). The area under the curve (AUC) for blood glucose following test drink consumption was $15 \%$ higher in the BE trial compared to BR $(P=0.006)$. Serum insulin AUC post-test drink tended to be greater in FR compared to all other trials $(P=0.07, P=0.124$ and $P=0.09$ vs. BR, FE and BE). There was a main effect of trial on NEFA concentrations $(P=0.002)$. GLP- $1_{7-36}$ concentrations were not significantly different between trials $(P=0.13)$. The AUC for hunger in response to ingestion of the test drink was greater when breakfast was omitted at rest (FR v BR: $P=0.018)$, which became a trend when exercise was performed (FE v BE: $P=0.053)$. No detectable difference was observed in lunch energy intake $(P=0.78)$. A main effect of trial was observed for postlunch energy, carbohydrate and fat balance (all $P<0.001$ ).

The influence of an acute bout of exercise on glucose tolerance is dependent upon prior nutritional state with no significant effect on insulin or GLP-1 $1_{7-36}$ concentrations. Exercise reduces the impact of breakfast consumption on feelings of hunger, yet subsequent energy intake is not significantly affected. Breakfast consumption at rest results in the most positive energy balance, with breakfast omission and exercise producing the least positive energy balance.

1. Astbury NM, Taylor MA \& Macdonald IA (2011) Breakfast consumption affects appetite, energy intake, and the metabolic and endocrine responses to foods consumed later in the day in male habitual breakfast eaters. Am J Clin Nutr 141, 1381-1389.

2. Borghouts LB \& Keizer HA (2000) Exercise and insulin sensitivity: a review. Int J Sports Med 21, 1-12.

3. Broom DR, Batterham RL, King JA et al. (2009) Influence of resistance and aerobic exercise on hunger, circulating levels of acylated ghrelin, and peptide YY in healthy males. Am J Physiol Regul Inter Comp Physiol 296, R29-R35. 